Laser Chem. 1988, Vol. 9, pp. 385-400

(C) 1988 Harwood Academic Publishers GmbH

Photocopying permitted by license only

Reprints available directly from the Publisher

Printed in the United Kingdom

\title{
Rotational Relaxation of Benzophenone in the Vapour Phase
}

\author{
M. A. STRAHAND, B. PEARCE and G. S. BEDDARDt \\ Department of Chemistry, University of Manchester, Manchester M13 9PL
}

(Received 20 May, 1988)

The excited singlet state lifetimes of benzophenone vapour have been measured at shorter wavelengths than previous studies and with picosecond time resolution. Excitation was at a series of wavelengths from $313.5 \mathrm{~nm}$ to $284 \mathrm{~nm}$, which gave $S_{2}\left(\pi \pi^{*}\right)$ decay times of tens of picoseconds. We also report measurements of the polarisation anisotropy decay due to free rotation of excited state benzophenone. Time-dependent anisotropies calculated by both quantum mechanical and classical formulae are in good agreement with the experimental data and demonstrate benzophenone to behave as a rigid rotor. From the residual anisotropy, at long times, it is concluded that no rotation to vibrational energy transfer occurs during the excited state lifetime.

KEY WORDS: Benzophenone vapour, picosecond, rigid rotor.

\section{INTRODUCTION}

Benzophenone has been the subject of many experimental studies, both in the gas phase and in solution. In solution the photophysics is governed by a rapid $\left(8 \rightarrow 30 \mathrm{ps}^{-1}\right) S_{1} \rightarrow T$ intersystem crossing ${ }^{1}$ rate and almost complete lack of fluorescence from the $n \pi^{*} S_{1}$ state. In the isolated molecule limit, gas phase benzophenone has a long luminescence lifetime, of some hundreds of nanoseconds, and which depends upon excitation wavelength. Because of this long lifetime, the excited molecules can collide with the cell walls or drift out of the detection region unless special precautions are taken, and these effects have led 
to the observation of complex ${ }^{2,3}$ and biexponential decay profiles. ${ }^{4}$ The longest of these decays yielded lifetimes greatly in excess of the natural radiative lifetime of the state excited, and this was explained by extensive singlet-triplet mixing. ${ }^{4}$ Naaman $e t$ al..$^{5}$ used an experimental arrangement which removed the possibility of wall collisions or drift out of the detector region. They generated an effusive beam of benzophenone, detected the emission by observing along and perpendicularly to the beam direction, and measured only single exponential decays when the benzophenone was isolated in its excited state. The $S_{1}$ lifetimes measured varied with excitation energy from $470 \mathrm{~ns}$ at $337 \mathrm{~nm}$ to $690 \mathrm{~ns}$ at $367 \mathrm{~nm}$, and a very large collision cross section was claimed to be responsible for the longer lifetimes previously reported. Besides the early work of Borisevich et al. ${ }^{6}$ who measured a $6.5 \mathrm{~ns}$ duration emission exciting into the $\pi \pi^{*}$ absorption, no other similar experiments have been reported exciting into $S_{2}$. In an effort to understand the excited state properties of benzophenone we have extended the range of measurements to shorter wavelengths than used previously and also down to the picosecond region.

\section{EXPERIMENTAL}

Excited state decays were measured using a pump/probe technique with multiphoton ionisation detection. ${ }^{7}$ Subpicosecond pulses $(0.8 \mathrm{ps}$ fwhm autocorrelation) originate from a synchronously pumped and mode-locked dye laser with an intracavity saturable absorber. ${ }^{8}$ The pulses were amplified to $800 \mu \mathrm{J}$ per pulse in a four stage dye laser amplifier pumped by a Nd:YAG laser operating at $10 \mathrm{~Hz}$. The experimental arrangement is as shown in Figure 1. The amplified pulse was split, $60 \%$ telescoped, frequency-doubled and the fundamental filtered out. The U.V. was passed through a calcite polariser. The visible beam, transmitted by the beam splitter, was passed along a delay line and its polarisation rotated either parallel or perpendicular to the pump beam. The U.V. and visible beams were recombined at the sample by a Pellin-Broca prism and focussed with the same $300 \mathrm{~mm} \mathrm{fl}$ lens. A photodiode after the cell monitored the U.V. and visible reference signal levels. The ion signal was measured by a charge sensitive pre-amplifier and further amplified (Ortec 575) before being digitised by a fast track and hold and ADC circuit. Ion signals were 


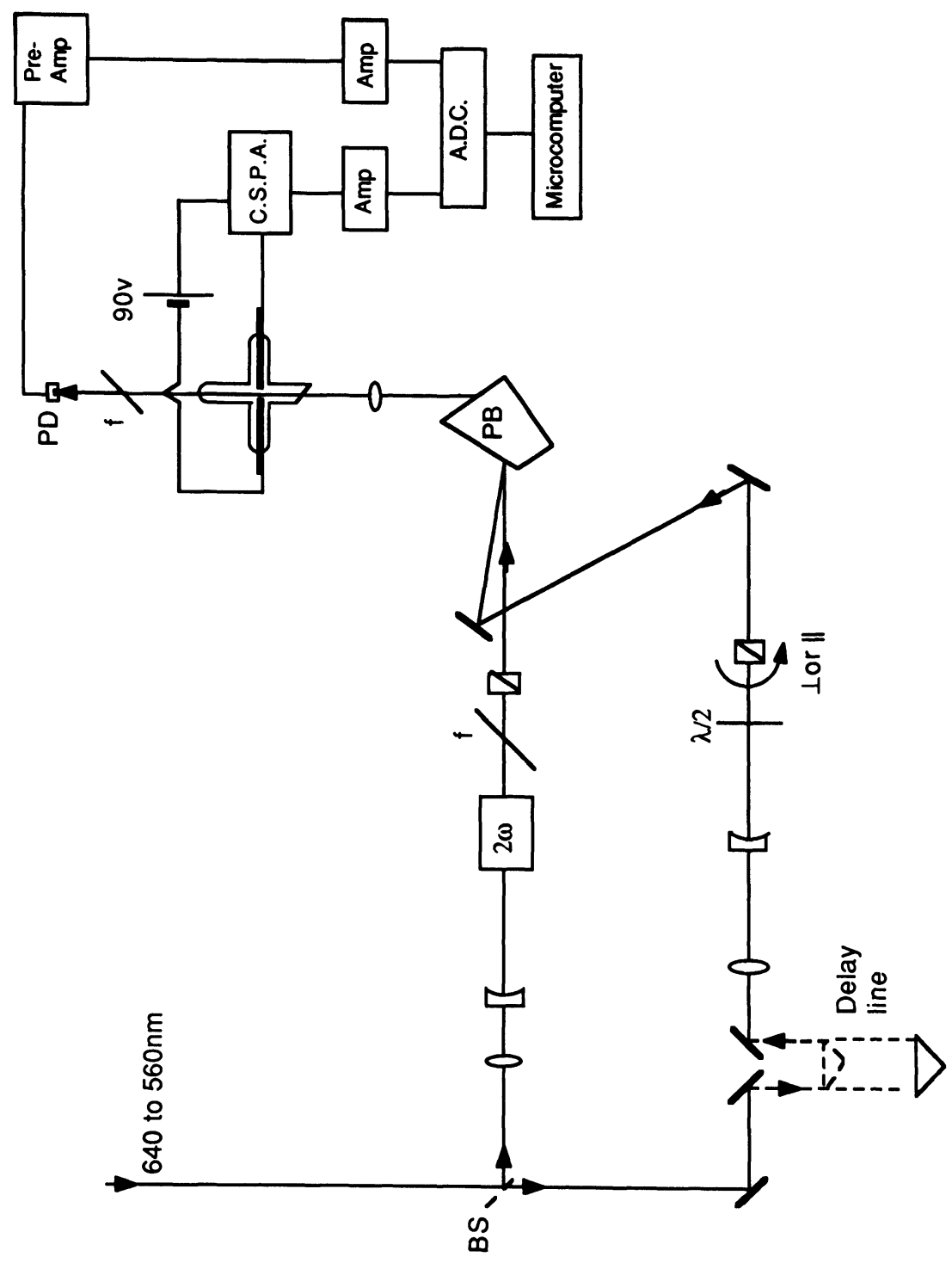

若密安

을 缃: a

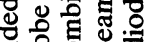
명 8 은 ज园

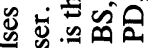
ล

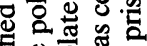
a 을

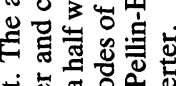
年 政 政

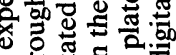

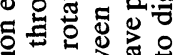
政 政 음의 0 영

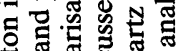

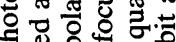
워워 当 웅

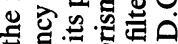
ot 0

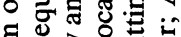
园远青

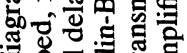
음

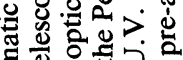
\% . 园 - \&> จ

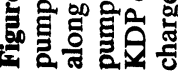




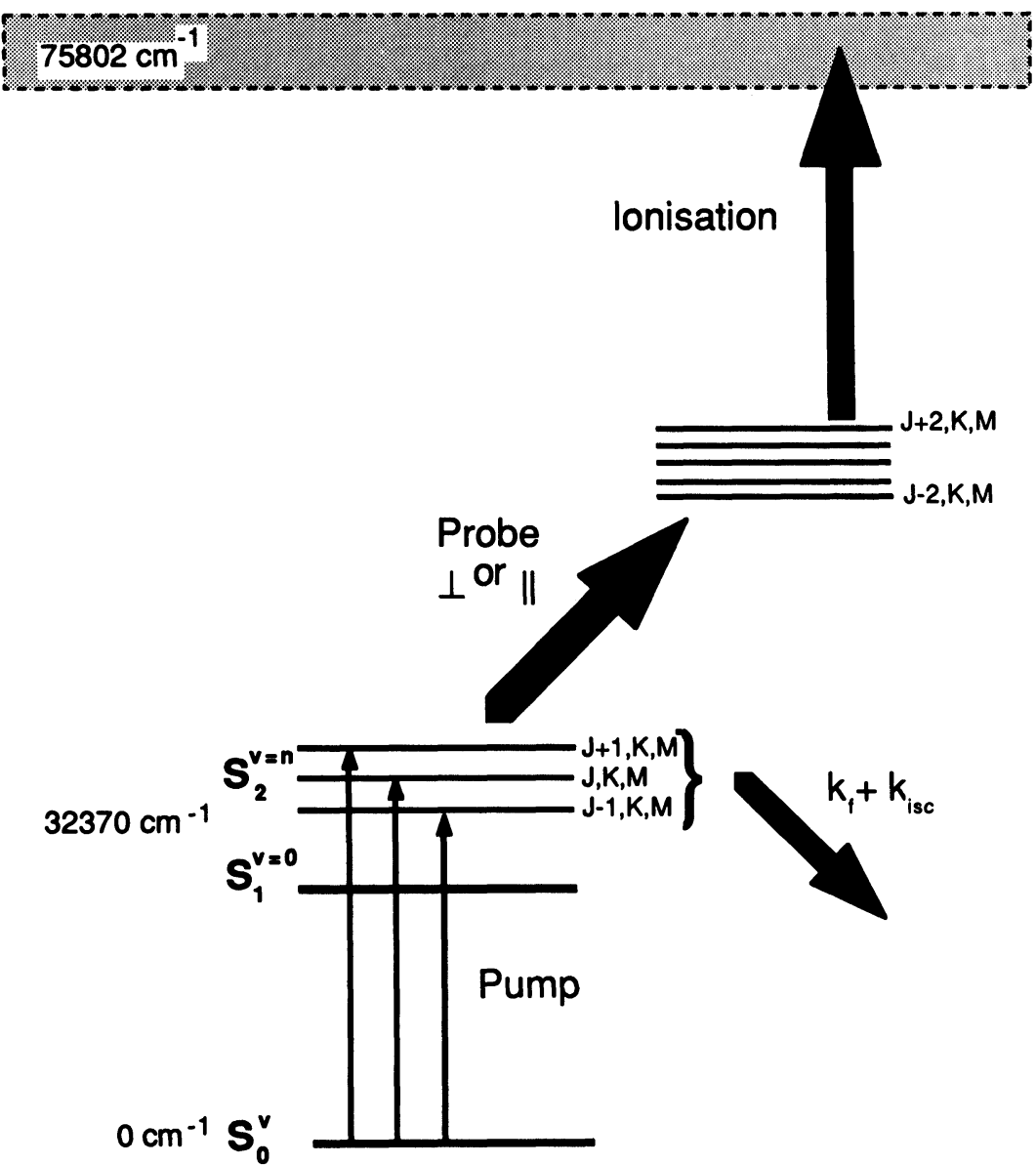

Figure 2 Benzophenone energy levels. A set of rotational levels in the $S_{2}$ vibrational manifold are coherently excited by the pump pulse and are ionised by the probe pulse. An intermediate set of rotational levels is also shown.

normalised for laser intensity fluctuations and poor pulses discriminated against by passing the reference signal through a narrow window of a single channel analyser. The cell contained benzophenone vapour in equilibrium with the solid which was contained in a side arm. Measurements were made at $294 \mathrm{~K}$, and at this temperature the vapour pressure was estimated to be $4 \times 10^{-4}$ torr, ${ }^{9}$ which ensured 
isolated molecule conditions during the time-scale of the experiment. Laser wavelengths were calibrated against optogalvanic lines in a neon discharge.

The ionisation energy of benzophenone is $75802 \mathrm{~cm}^{-1} \cdot{ }^{10}$ Using only fundamental $\left(\omega_{1}\right)$ and frequency doubled $\left(\omega_{2}\right)$ light, photons of wavelength shorter than $659.6 \mathrm{~nm}$ are required to ionise the $a_{1}$ state in a four photon $\left(\omega_{2}+3 \omega_{1}\right)$ scheme, Figure 2 . The ion signal diminishes in proportion to the number of excited molecules present, and thus measures the excited state decay. ${ }^{7}$ The intensity of both the excitation and ionising beams was carefully controlled so as to prevent ionisation from either beam alone. Some ionisation from the excitation beam alone is, however, unavoidable and contributes a constant background signal. A five to eight times ion signal enhancement is observed when both beams are present compared to the excitation beam alone, Figure 3a.

\section{RESULTS AND DISCUSSION}

The absorption spectrum of benzophenone shows a broad and weak $n \pi^{*}$ transition centered at $350 \mathrm{~nm}$ and more intense and structureless absorption starting at about $300 \mathrm{~nm}$ and peaking at $255 \mathrm{~nm}$ which is due to $\pi \pi^{*}$ transitions. Excitation into the $n \pi^{*}$ state produces a long lived emission, which decays at a rate of $1.5 \times 10^{6}$ to $2.13 \times 10^{4} \mathrm{~s}^{-1}$, depending on excitation energy. ${ }^{5}$ The radiative rate of this state as measured from the integrated absorption spectrum is $0.5 \times 10^{6} \mathrm{~s}^{-1}$. It has been suggested ${ }^{5}$ that benzophenone is in the large molecule statistical limit, and that the increase in rate with excess energy in $S_{1}$ is due to $S_{1} \rightarrow S_{0}$ internal conversion. Wall quenching and a very large collisional cross section for intersystem crossing were used to explain the observation of hot and then red shifted phosphorescence with a millisecond decay time. ${ }^{3,5}$

The $\pi \pi^{*}$ transitions have been analysed by Yoshino et al. ${ }^{11}$ based on reference wavefunctions of a benzene dimer and a carbonyl group. Using polarised absorption in stretched films they identified transitions at 317,305 and $257 \mathrm{~nm}$. The $257 \mathrm{~nm}, A_{1} \rightarrow B_{2}$, transition is about seven times more intense than the other two $A_{1} \rightarrow A_{1}$ transitions. The location of these weak transitions must be uncertain, however, as they overlap extensively and generate only small changes in the polarisation 


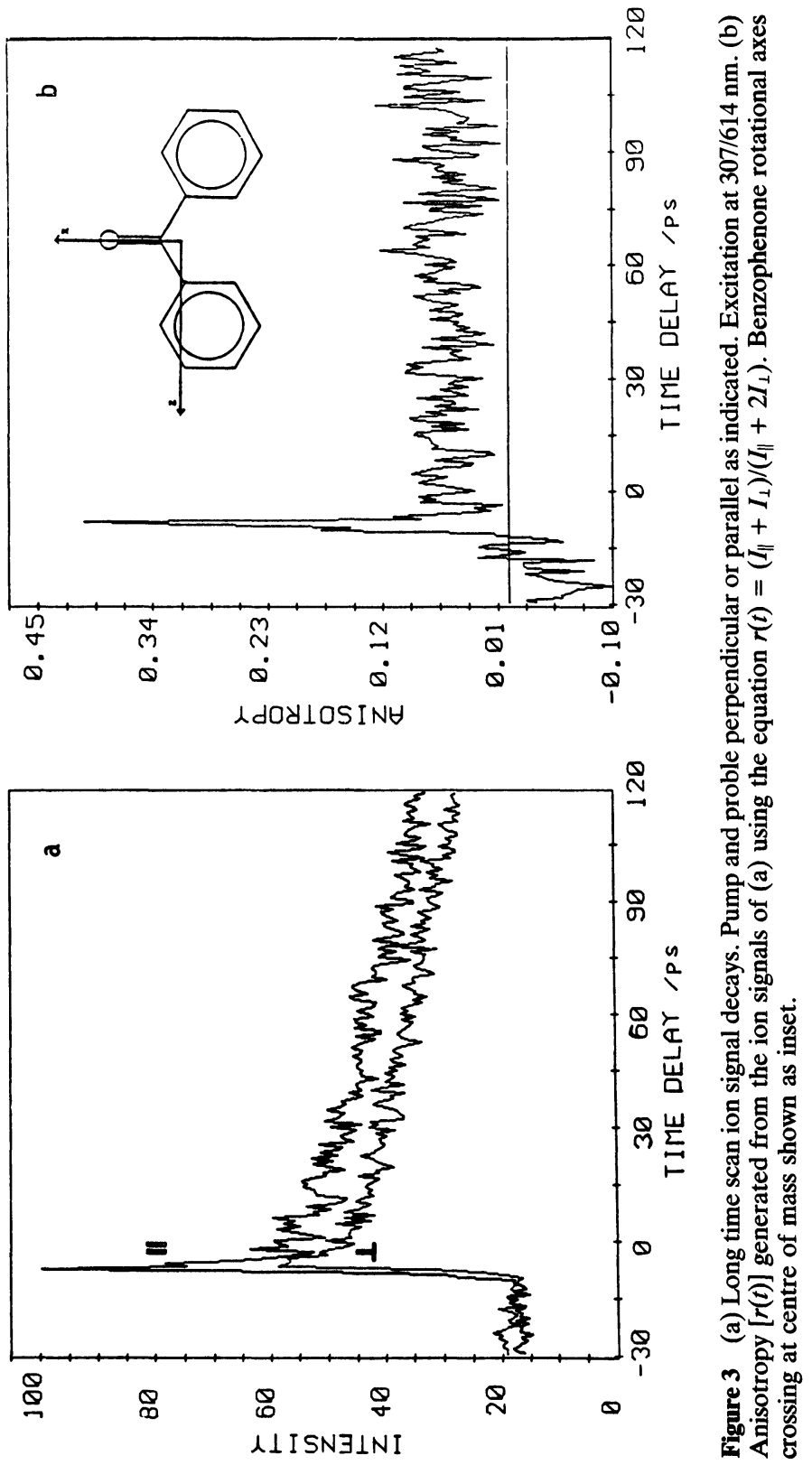


ratio of stretched films. In our experiments we excited into the tail of the $257 \mathrm{~nm}$ transition and also into the short wavelength end of the $n \pi^{*}$ transition. Table I lists the decay times observed exciting from 313.5 to $284 \mathrm{~nm}$. At the longest two wavelengths very small signals were observed and we attribute this as due to excitation primarily into the $n \pi^{*}$ absorption from which we assume that the overall ionisation cross section is small compared to that of the $\pi \pi^{*}$ state. At shorter wavelengths the excited state lifetime was measured as several tens of picoseconds, Table I, and this great shortening compared to the $n \pi^{*}$ state is probably due to spin-orbit enhanced $\pi \pi^{*} \rightarrow T_{n}$ intersystem crossing. The radiative rate of the $\pi \pi^{*}$ state is not known but if we assume that it is similar to that of benzene $\left(2.40 \times 10^{6} \mathrm{~s}^{-1}\right)$ the reciprocal fluorescence lifetime is effectively the non-radiative rate constant. The decrease in lifetime with increasing energy in the excited state is typical of many aromatic molecules and in general terms is explained by radiationless transition theory as an increase in the Franck-Condon weighted density of states between the two states involved. ${ }^{12}$ The benzophenone absorption spectrum is very congested, it is unknown which levels are being excited and thus it is unclear how to apply the Golden rule formula directly. The approach of Jacobson $e t$ $a l .{ }^{13}$ in treating high vibrational levels in substituted naphthalenes should be of more use. They show that the non-radiative rate depends on the ratio of the density of states times the square of the average interaction matrix element when many initial levels are excited. Using this approach, calculating the density of states using Raman and i.r.

Table I Excited state lifetimes and radiation times of benzophenone vapour

\begin{tabular}{lll}
\hline Wavelength & Decay time $(\mathrm{ps})$ & ${\text { Rotation time }( \pm 0.5 \mathrm{ps})^{\mathrm{b}}}^{\mathrm{b}}$ \\
\hline 284 & 66 & 1.0 \\
290 & 100 & 1.0 \\
301.5 & 113 & 1.5 \\
307 & 112 & 1.7 \\
310.5 & $\mathrm{a}$ & $\mathrm{a}$ \\
313.5 & $\mathrm{a}$ & $\mathrm{a}$ \\
\hline
\end{tabular}

\footnotetext{
${ }^{a}$ No ion signal enhancement.

b Anisotropy decay assumed exponential in non-linear least squares convolution with instrument profile.
} 
frequencies, ${ }^{14}$ leads to a $1.7 \times$ increase in rate between 307 and $284 \mathrm{~nm}$ which is of the correct order of magnitude increase as shown by the data. The larger non-radiative decay rate from the $\pi \pi^{*}$ than the $n \pi^{*}$ state could be due to allowed ${ }^{1} \pi \pi^{*} \rightarrow{ }^{3} n \pi^{*}$ crossing, via a spin-orbit coupling operator with $B_{1}$ symmetry (in $C_{2 v}$ ), compared to the "forbidden" ${ }^{1} n \pi^{*} \rightarrow{ }^{3} n \pi^{*}$ transfer.

When the polarisation of the pump and probe are parallel a rapidly decaying transient is observed at short times after excitation, Figure 3 and we attribute this transient to the free rotor motion of the excited molecule. Similar transients were observed from 4,4'-dimethoxy and 4,4'-dichlorobenzophenone. Stilbene also exhibits polarisation dependent signals, and our measurements are similar to those of Zewail et al., ${ }^{7}$ but in aniline and several substituted naphthalenes no fast transient on the decay of the ionisation signal was observed.

The data in Figure 3a shows the effect of probe polarisation both parallel and perpendicular to the excitation, from this data the anisotropy $r(t)=\left[I_{\|}(t)-I_{\perp}(t)\right] /\left[I_{\|}(t)+I_{\perp}(t)\right]$ was calculated, Figure $3 \mathrm{~b}$ where $I_{\|}$and $I_{\perp}$ are signals at time $t$ for probe polarisation parallel and perpendicular to the pump polarisation respectively. Initially the anisotropy decays on the time scale of $(I / k T)^{1 / 2}$ where $I$ is the moment of inertia, but at longer times does not fall to zero but is a slowly varying function of the moments of inertia. ${ }^{15}$ At very long times, or increased pressures, collisions will change the angular momentum of the molecule and the anisotropy should tend to zero, as in solution. At short times the free rotor behaviour should still be observable. This transient, which represents the rigid-body rotational motion in a classical picture, is in quantum terms due to interferences between different intermediate $J$ levels that are connected to the same initial and final states, Figure 2. Using a quantum approach Felker et al. ${ }^{16}$ have examined fluorescence transients produced from free rotation in isolated molecules, this approach can be extended to describe ionisation experiments if we assume that ionisation occurs equally well from any of the intermediate $J$ levels produced, Figure 2.

When a particular vibronic state and rotational level $\left(J_{0} K_{0} M_{0}\right)$ is excited by a short pulse of polarised light a coherent superposition of rotational levels $\phi(t)$, is produced in the excited vibronic manifold. This superposition evolves in time as transitions occur by fluorescence or absorption to the final state $\phi(J K M)_{f}$. The signal intensity with 
either parallel or perpendicular pump and probe polarisations, can be calculated after averaging over a thermal distribution of $J_{0}$ and $K_{0}$,

$$
\begin{aligned}
& I(J, K)=\sum_{J K M_{f}}\left|\left\langle\phi(t)\left|e_{f} \cdot \mu\right| \phi(J, K, M)_{f}\right\rangle\right|^{2} \\
& I(t) \infty \sum_{J_{0} K_{0}} I(J, K) \exp \left(-E_{r} / K_{b} T\right)
\end{aligned}
$$

where $E_{r}=B J_{0}\left(J_{0}+1\right)+(A-B) K_{0}^{2}$ and $A$ and $B$ are the usual rotational constants of a prolate symmetric top; $e_{f}$ is the detection polarisation and $\mu$ the dipole moment operator. Finally $I(t)$ should be multiplied by the population decay probability exp $(-t / \tau)$.

If we assume that the benzophenone although asymmetric, with principal moments of inertia $\left(x 10^{-44} \mathrm{Kgm}^{2}\right) I z=0.49, I x=1.93$ and $I y$ $=2.42,{ }^{17}$ can be represented as a symmetric top and averaging $I x$ and $I y$, Eq. (1) may be evaluated with selection rules $\Delta J= \pm 1,0$ and $\Delta K=$ 0 for parallel transitions and $\Delta K= \pm 1$ for perpendicular transitions, and by summing over $M$ to account for the isotropy of the sample. The unique inertial axis lies perpendicular to the carbonyl bond and in the plane of the molecule. The $A_{1} \rightarrow B_{2}\left(\pi \pi^{*}\right)$ transition is polarised along this axis and the $n \pi^{*}$ transition is perpendicular to it. In evaluating Eq. (1) the direction-cosines are tabulated by Cross et al. ${ }^{18}$ and polarisation dependent factors for the time independent terms by Loge and Parmenter. ${ }^{19}$ Time dependent terms arise as cosines with frequencies $v_{1}=$ $2 B J_{0}, v_{2}=2 B\left(J_{0}+1\right)$ and $v_{3}=v_{1}+v_{2}$ when $\Delta K=0$. For $\Delta K= \pm 1, v_{1}$ $=2 B J_{0} \pm v_{k}, v_{2}=2 B\left(J_{0}+1\right) \pm v_{k}$, and $v_{3}=v_{1}+v_{2} \pm v_{k}$, where $v_{k}=(A$ $-B) K^{2}$. The factors needed to evaluate these off-diagonal terms are given in the appendix. Interference between the cosine terms generates the initial oscillatory nature of the anisotropy near $t=0$, reoccurrences may also occur at longer times. ${ }^{16}$ Figure 4 shows transients calculated using Eq. (1).

As described above no transients attributable to rotational motion were observed in aniline or several naphthalenes for reasons that are unclear. The lack of fast signals could mean that rotational to vibrational coupling is fast, but we have excited both below and above the energies expected for the onset of IVR with similar results. Another possibility is that the pumping or ionising transition is easily saturated in these molecules where the signals are several hundred times greater than from benzophenones. In saturation the laser would excite most molecules in the cosine squared distribution about the direction of 


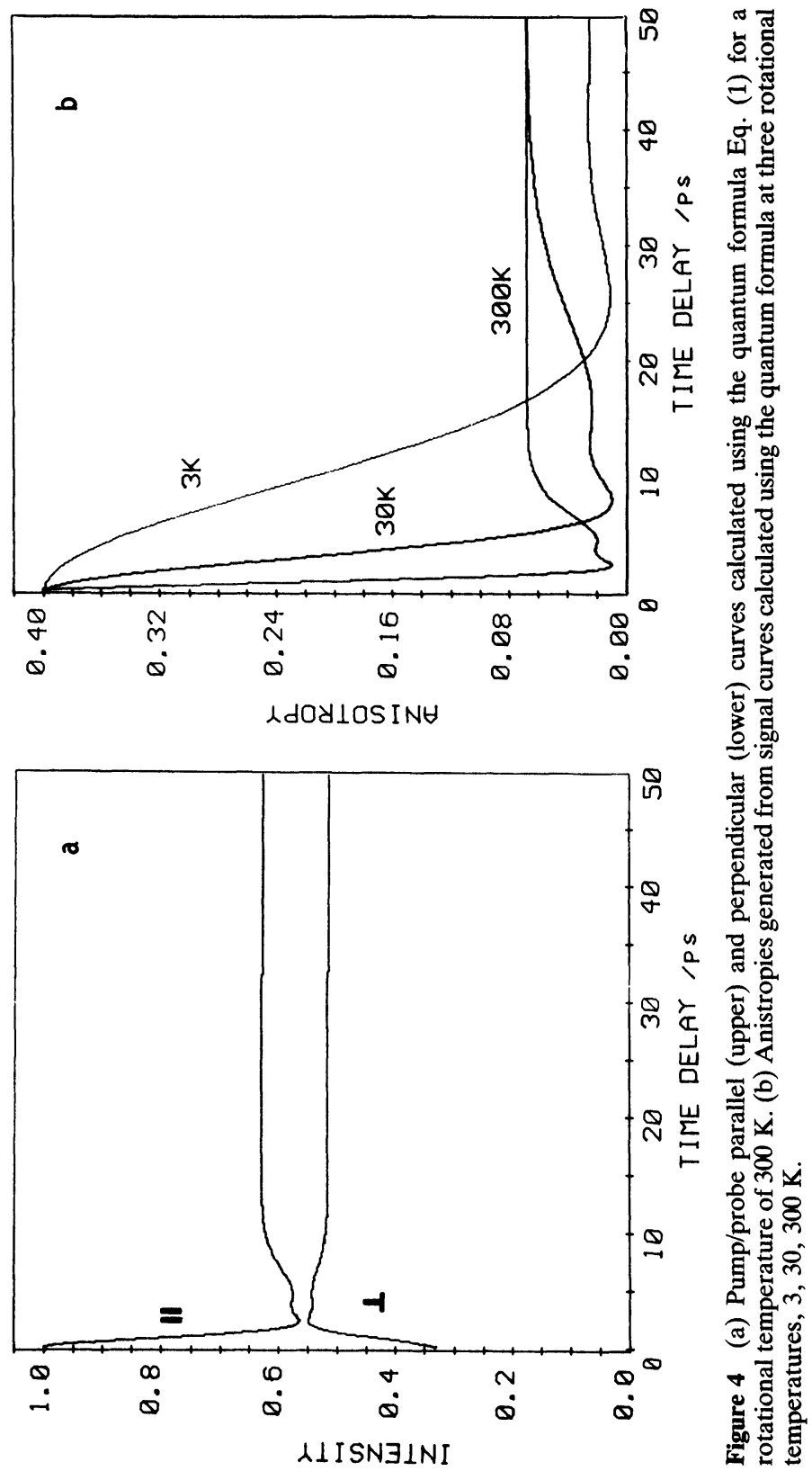


laser polarisation $;^{21}$ this would produce a more isotropic distribution and remove the possibility of observing rotational motion.

Because our experiments were done at room temperature many $J$ levels ( 430) need to be summed over in evaluating Eq. (1), and both $I_{\|}(t)$ and $I \perp(t)$ calculated to obtain $r(t)$. Alternatively $r(t)$ can be calculated directly [but not $I(t)$ ] from the classical formulae of Yang and Bersohn ${ }^{20}$ vis,

$$
r(t)=g_{00}\left\langle D_{00}(t)\right\rangle+g_{11}(t)\left\langle D_{11}(t)\right\rangle+g_{22}\left\langle D_{22}(t)\right\rangle
$$

where the $g$ 's are sums of products of spherical harmonics, and describe the angle between the absorption, emission (probe) dipole moments and the principal axis. The $D$ 's are the correlation functions averaged over reorientation angles.

These functions are simpler to evaluate than the equivalent quantum ones and surprisingly we found that for benzophenone even at as low a temperature as $3 K$ the anisotropy calculated from the classical model is almost indistinguishable from that calculated by the quantum formulae. The reason being the large number of $J$ levels are still populated at this temperature. Convoluting the calculated anisotropy with a Gaussian pulse of the duration of our laser pulse produces similar decay products to those measured experimentally, Figure 5. The decay of the calculated $r(t)$ gave a lifetime very similar to the $\sim 1.5$ ps measured experimentally.

The long time value for the anisotropy $r(\infty)$ is $0.06 \pm 0.02$ and compares well with the calculated value of 0.067 for a parallel transition. If the transition were perpendicular to the unique inertial axis (i.e. $n \pi^{*}$ state) $r(\infty)$ would be $1 / 4$ that of the parallel case. ${ }^{15,21}$ In principle the measured $r(\infty)$ value could indicate which state the excited singlet decays into. If the ${ }^{3} n \pi^{*}$, or another state which is perpendicularly polarised is populated, $r(\infty)$ should decrease, however, in practice since the ion signals $[I(t)]$ return to close to the background level the cross-section for ionisation from the ${ }^{3} n \pi^{*}$ state must be small compared to that from the initially excited state. The initial anisotropy value $r(0)=0.4 \pm 0.05$ is effectively at its maximum value which means that the dipoles for absorption and ionisation are parallel and that little if any rotation to vibrational coupling occurs on the fast time scale i.e. during the laser pulse. Similarly the value of $r(\infty)$ implies that in benzophenone hundreds of rotational periods are needed before rotational to vibrational energy transfer occurs. If some rotation to 


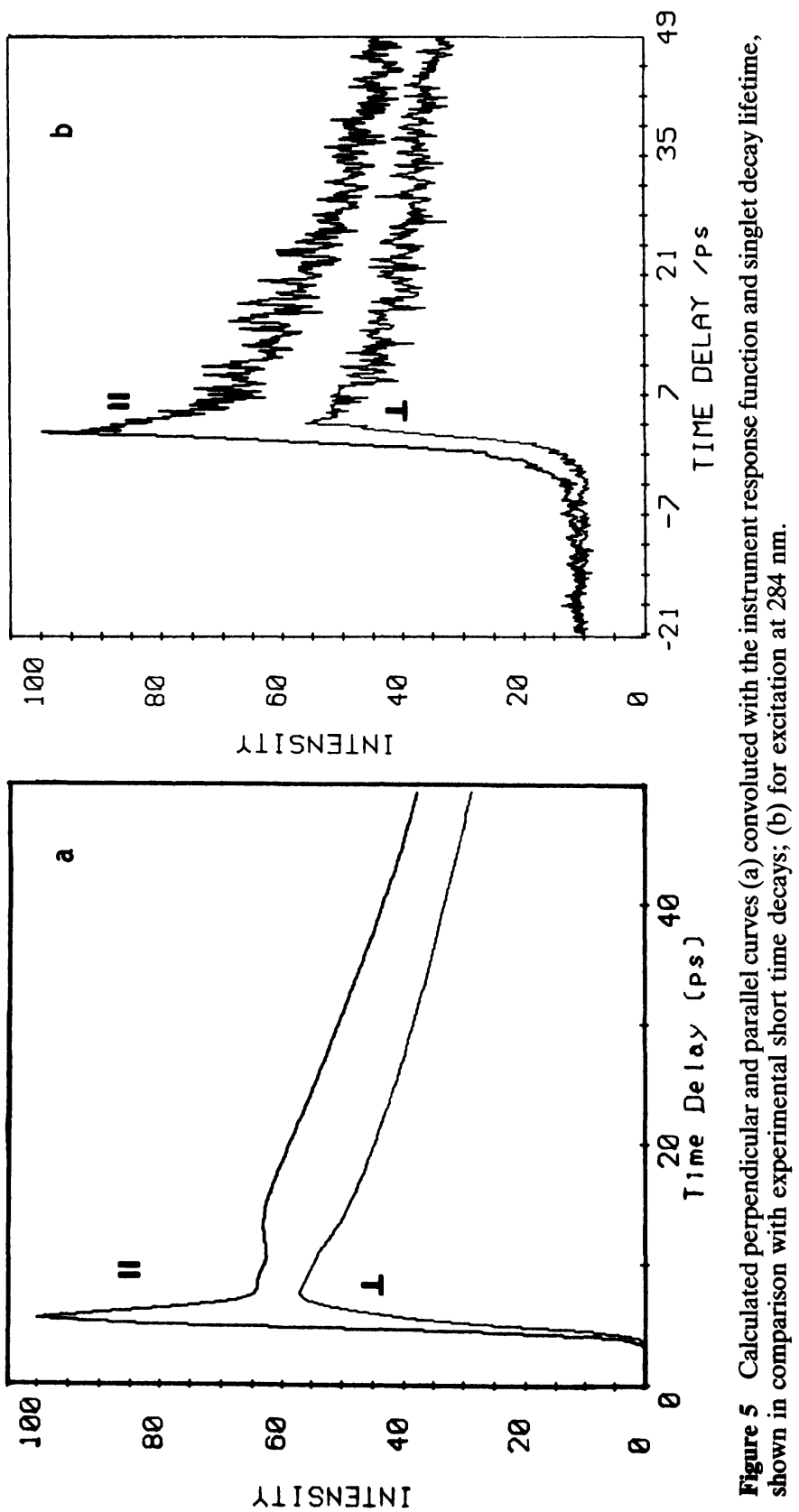


vibrational coupling were occurring, as is the case in stilbene where $r(0)=0.15-0.2$ and $r(\infty)=0.05,{ }^{22}$ or pyrimidine ${ }^{23}$ the $r(0)$ value for benzophenone would be smaller than measured and the $r(\infty)$ could decrease to 0.034 which is the limit for complete statistical behaviour in a parallel transition. ${ }^{15}$

\section{Acknowledgements}

This work was supported from grants from the Royal Society and the SERC.

\section{References}

1. R. W. Anderson, R. M. Hochstrasser, H. Lutz and G. W. Scott, Chem. Phys. Lett. 28, 153 (1974).

2. G. E. Busch, P. M. Rentzepis and J. Jortner, Chem. Phys. Lett. 11, 437 (1971).

3. G. E. Busch, P. M. Rentzepis and J. Jortner, J. Chem. Phys. 56, 361 (1972); D. Zevenhuijzen and R. Van der Werf, Chem. Phys. 26, 279 (1977).

4. R. M. Hochstrasser and J. E. Wessel, Chem. Phys. Lett. 19, 156 (1973).

5. R. Naaman, D. M. Lubman and R. N. Zare, Chem. Phys. 32, 17 (1987).

6. N. A. Borisevich and V. V. Grunzinskii, Doklady Akad. Nauk. SSSR 175, 852 (1967).

7. J. W. Perry, N. F. Scherer and A. H. Zewail, Chem. Phys. Lett. 103, 1 (1967).

8. R. J. Harrison, B. Pearce, G. S. Beddard, J. A. Cowan and J. K. M. Sanders, Chem. Phys. 116, 429 (1987).

9. C. R. C. Handbook of Chemistry and Physics, 60th edition.

10. F. H. Field and J. Franklin, "Electron impact phenomena and the properties of gaseous ions" Academic 1970.

11. J. Yoshino, T. Hoshi, H. Ogawa, T. Minegishi and Y. Tanizaki, Berichte der Bunsen Gesellschaft 81, 748 (1977).

12. K. F. Freed, Topics Appl. Phys. 15, 23 (1976).

13. B. A. Jacobson, J. A. Guest, F. A. Novak and S. A. Rice, J. Chem. Phys. 87, 269 (1987).

14. C. Menard and A. Melier, Spectrochim. Acta 29A, 1273 (1973).

15. G. Nathanson and G. M. McLelland, J. Chem. Phys. 81, 629 (1984).

16. P. M. Felker, J. S. Baskin and A. H. Zewail, J. Phys. Chem. 90,724 (1986); P. M. Felker and A. H. Zewail, J. Chem. Phys. 86, 2460 (1987); J. S. Baskin, P. M. Felker and A. H. Zewail, J. Chem. Phys. 86, 2483 (1987).

17. E. B. Fleischer, N. Sung and S. Hawkinson, J. Phys. Chem. 72, 4311 (1968).

18. P. C. Cross, R. M. Hainer and G. W. King, J. Chem. Phys. 12, 210 (1944).

19. G. W. Loge and C. S. Parmenter, J. Chem. Phys. 74, 29 (1981).

20. J. H. Ling and K. R. Wilson, J. Chem. Phys. 65, 881 (1976).

21. S.-C. Yang and R. Bersohn, J. Chem. Phys. 61, 4400 (1974).

22. A. J. Bain, C. Han, P. L. Holt, P. M. McCarthy, A. B. Myers, M. A. Pereria and R. M. Hochstrasser in Ultrafast Phenomena IV, p. 489 (1986).

23. G. M. Nathanson and G. M. McLelland, J. Chem. Phys. 84, 3170 (1986); J. Chem. Phys. 85, 4311 (1986). 


\section{APPENDIX}

The polarisation dependent factors of the product of absorption transition probability and the probing transition probability averaged over the $2 J+1$ initial $M$ states, and used in the quantum calculation, are listed here. The absorption probing sequence is treated as a coupled process with a common $J^{\prime} K^{\prime} M^{\prime}$ rotational state in the excited electronic state. The same notation as Loge and Parmenter ${ }^{19}$ is used; $J$ ground state, $J^{\prime}$ excited electronic state and $J^{\prime \prime}$ final state, Figure 1 . The factors to be multiplied by time dependent terms have four indices as they arise from interference between two levels in the excited electronic state. The expressions should all be multiplied by $8(2 J+1) / 15$. 

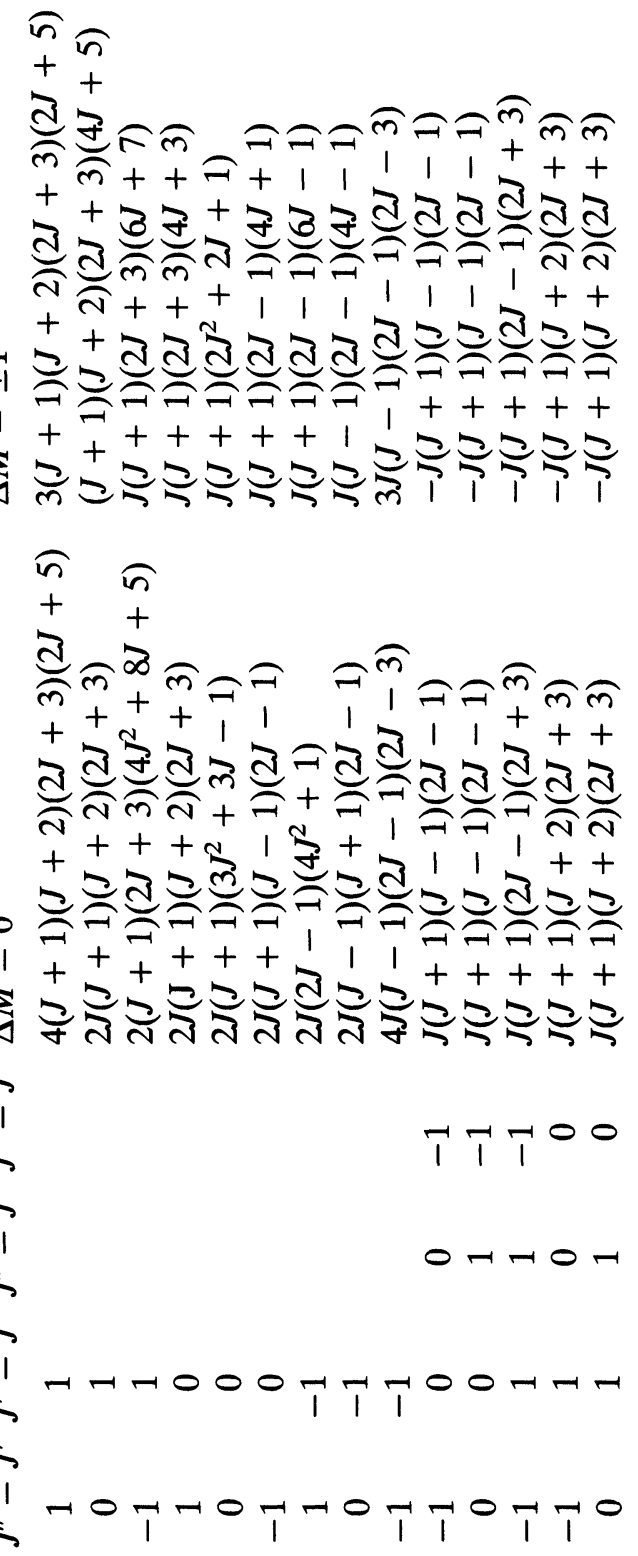
For a parallel transition $I(J, K)$ is given by the formula:

$$
I(J, K)=\alpha+\beta \cos \left(2 \pi v_{1} t\right)+\gamma \cos \left(2 \pi v_{2} t\right)+\delta \cos \left(2 \pi v_{3} t\right)
$$

When the pump and probe polarisations are parallel

$$
\begin{aligned}
\propto= & 2 / 15 \cdot\left(J^{2}-K^{2}\right) / J \cdot\left[\left((J-1)^{2}-K^{2}\right) /((2 J-1)(J-1)\right. \\
& \left.+K^{2}\left(J^{2}+1\right) /\left(J^{2}\left(J^{2}-1\right)\right)\right]+1 / 15 \cdot\left[K^{4}(2 J+1)\left(3 J^{2}+3 J-1\right) /\right. \\
& \left(J^{3}(J+1)^{3}\right)+\left(J^{2}-K^{2}\right)^{2}\left(4 J^{2}+1\right) /\left(J^{3}\left(4 J^{2}-1\right)\right) \\
& \left.+\left((J+1)^{2}-K^{2}\right)^{2}\left(4 J^{2}+8 J+5\right) /\left((J+1)^{3}(2 J+1)(2 J+3)\right)\right] \\
& \left.+2 / 15 \cdot\left((J+1)^{2}-K^{2}\right) /(J+1)(J+2)\right) \cdot\left[K^{2}\left(J^{2}+2 J+2\right) /\right. \\
& \left.\left(J(J+1)^{2}\right)+\left((J+2)^{2}-K^{2}\right) /(2 J+3)\right]
\end{aligned}
$$

and when perpendicular;

$$
\begin{aligned}
& \propto= 1 / 15 \cdot\left(J^{2}-K^{2}\right) / J \cdot\left[3 / 2\left((J-1)^{2}-K^{2}\right) /((2 J-1)(J-1)\right. \\
&\left.+K^{2}\left(4 J^{2}-1\right) /\left(J^{2}\left(J^{2}-1\right)\right)\right] \\
&+1 / 15 /\left[K^{4}(2 J+1)\left(2 J^{2}+2 J+1\right) /\left(2 J^{3}(J+1)^{3}\right)\right. \\
&+\left(J^{2}-K^{2}\right)^{2}(J+1)(6 J-1) /\left(2 J^{3}\left(4 J^{2}-1\right)\right)+\left((J+1)^{2}\right. \\
&\left.\left.-K^{2}\right)^{2} J(6 J+7) /\left(2(J+1)^{3}(2 J+1)(2 J+3)\right)\right] \\
&+1 / 15 \cdot\left((J+1)^{2}-K^{2}\right) /((J+1)(J+2)) \cdot \\
& {\left[K^{2}(2 J+1)(2 J+3) /\left(J(J+1)^{2}\right)+3 / 2\left((J+2)^{2}-K^{2}\right) /(2 J+3)\right] }
\end{aligned}
$$

and $\beta=2 P / 15 \cdot K^{2}\left(J^{2}-K^{2}\right) /\left(J^{2}(J+1)\right)$;

$$
\begin{aligned}
& \gamma=2 P / 15 \cdot K^{2}\left((J+1)^{2}-K^{2}\right) /\left(J^{2}(J+1)^{2}\right) \\
& \delta=2 P / 15 \cdot\left((J+1)^{2}-K^{2}\right)\left(J^{2}-K^{2}\right) /(J(J+1)(2 J+1))
\end{aligned}
$$

$P=2$ for parallel pump and probe polarisations and -1 for perpendicular probe polarisation. For a perpendicular transition the Eqs. $I(J, K)$ contain 75 terms each rather than the 15 for the parallel transition case, slightly simplfied, and reproduced above. 